\title{
Optimal Regularity Results in Boundary Control of Elastic Systems with Fractional Order Damping
}

\author{
Scott W. Hansen*
}

\begin{abstract}
In this article a criterion known as the "operator Carleson measure criterion" is applied to obtain regularity results for a general class of second-order boundary control problems. As specific examples, optimal regularity results are obtained for a wave equation and a plate equation, both with fractional order damping and on a rectangular domain with one edge controlled.
\end{abstract}

\section{Introduction and statement of the main results}

We consider the second-order control system

$$
w_{t t}+2 \gamma A^{\alpha} w_{t}+A w=B f
$$

where $A$ is a positive operator which is an isomorphism $Y_{1} \rightarrow Y_{0}$, for appropriately defined Hilbert spaces $Y_{1} \subset Y_{0}$. The damping operator $A^{\alpha}, \alpha \geq 0$, is well-defined by the spectral theorem and is an isomorphism $Y_{\alpha} \rightarrow Y_{0}$, where $Y_{\alpha}$ is an appropriately defined intermediate space. We wish to consider a class of $B$ operators motivated by boundary control problems. Thus $B$ may be "unbounded" in the sense that its range lies in a larger space than $Y_{0}$. We assume the minimal condition that $B$ is continuous from a Hilbert space $U$ to $Y_{-1}=Y_{1}^{\prime}$. The input $f$ is assumed to belong to the class $L^{2}(0, \infty ; U)$.

We are concerned with the problem of determining the optimal regularity of solutions of such control systems as a function of $\alpha$ for damping exponents $\alpha$ in the range $0<\alpha<1 / 2$. Since $B$ maps continuously into $Y_{-1}$, mild solutions with zero initial data

$$
w(0)=w_{t}(0)=0
$$

a-priori possess the regularity

$$
w \in C\left([0, T] ; Y_{-1 / 2}\right) \cap C^{1}\left([0, T] ; Y_{-1}\right), \quad \forall T>0 .
$$

If in addition, for some $\sigma \geq-1 / 2$

$$
W \in C\left([0, T] ; Y_{\sigma}\right) \cap C^{1}\left([0, T] ; Y_{\sigma-1 / 2}\right), \quad \forall T>0,
$$

\footnotetext{
${ }^{*}$ Department of Mathematics, Iowa State University, Ames, Iowa 50011
} 
then $B$ is called admissible relative to the state space $Y_{\sigma} \times Y_{\sigma-1 / 2}$. The problem is to determine the optimal $\sigma$ for which $B$ is admissible.

Our approach is to first transform the above system to an equivalent first order, diagonal system on $l^{2}$, where $A$ and $B$ are represented by infinite matrices. We then apply the operator Carleson measure criterion (OCMC) of Hansen and Weiss [5] [6], which provides a sharp criterion for the admissibility for such systems.

We consider two main examples. Both concern boundary control of elastic systems with fractional order damping on rectangular domain $\Omega$.

Example 1 First, consider the problem of a wave equation with fractional order damping subject to Dirichlet control on the boundary:

$$
\begin{cases}w_{t t}+2 \gamma(-\Delta)^{\alpha} w_{t}-\Delta w=0, & \text { in } \Omega \times \mathbb{R}^{+} \\ w=f & \text { on } \Gamma \times \mathbb{R}^{+}\end{cases}
$$

where $\Gamma=\delta \Omega$. We will see that the optimal regularity space for solutions to (1.2), (1.3), with $0 \leq \alpha<1 / 4$ is $L^{2}(\Omega) \times H^{-1}(\Omega)$; i.e., over the class of controls $f \in$ $L^{2}((0, \infty) \times \Gamma)$ one has the optimal regularity

$$
w \in C\left([0, \infty), L^{2}(\Omega)\right) \cap C^{1}\left([0, \infty), H^{-1}(\Omega)\right) .
$$

The regularity in (1.4) coincides with known optimal regularity results for the undamped case (with $\gamma=0$ ) obtained (in more generality) by Lasiecka, Lions and Triggiani, [9]. In this case the addition of fractional order damping does not change the optimal spaces.

Example 2 As our second example we consider a plate equation also with fractional order damping on a rectangular domain, but with moment control on the boundary:

$$
\begin{cases}w_{t t}+2 \gamma(-\Delta)^{2 \alpha} w_{t}+\Delta^{2} w=0, & \text { in } \Omega \times \mathbb{R}^{+} \\ w=0, \quad \frac{\partial^{2} w}{\partial n^{2}}=f & \text { on } \Gamma \times \mathbb{R}^{+}\end{cases}
$$

where $\Gamma=\partial \Omega$ and $n$ is the outward normal direction vector. In the case $\alpha=1$, the damping present in (1.5) is known as Kelvin-Voigt damping, while in the case $\alpha=1 / 2$ the damping model has been referred to as "square-root damping" or "structural damping"; see [1]. In the case $0 \leq \alpha \leq 1 / 2$ we obtain the optimal regularity result (for $f \in L^{2}((0, \infty) \times \Gamma)$ ):

$$
w \in C\left([0, \infty), X_{\widehat{a}+1}\right) \cap C^{1}\left([0, \infty), X_{\widehat{a}}\right),
$$

where $X_{0}=L^{2}(\Omega), X_{1}=H^{2}(\Omega) \cap H_{0}^{1}(\Omega)$ and $\widehat{a}=\min \{-1 / 4, \alpha-1 / 2\}$.

The regularity in (1.6) coincides with known results (see e.g., Lasiecka and Triggiani [10]) for the undamped case if $\alpha=0$ where one has admissibility in the space $H_{0}^{1}(\Omega) \times H^{-1}(\Omega)$ ). However as $\alpha$ is increased from zero to $1 / 4$, the corresponding space of admissibility of (1.6) also increases to $\left(H^{3 / 2}(\Omega) \cap H_{0}^{1}(\Omega)\right) \times H^{-1 / 2}(\Omega)$. For $1 / 4 \leq \alpha<1 / 2$ there is no increase in regularity. As $\alpha \rightarrow 1 / 2$ the regularity agrees with the regularity obtained by Triggiani [12] for the case $\alpha=1 / 2$. 
Regarding the background of this problem, Chen and Russell [1] proved that systems of the form (1.1) for $\alpha=1 / 2$ are described by an analytic semigroup while the subsequent work of S. Chen and Triggiani [2-4] showed that the analyticity remains for $1 / 2 \leq \alpha \leq 1$, but not for $0 \leq \alpha<1 / 2$. While this work did not specifically address boundary control, Triggiani [12] later obtained optimal regularity results for the case of boundary control as in Example 2 with $\alpha=1 / 2$, as mentioned above.

The approach here, in contrast to direct estimates of the previously mentioned articles, utilizes the OCMC (described in Section 2) to obtain the regularity results. A disadvantage of this approach is that one needs to calculate the matrix form of the $B$ operator in (1.1). However, if this can be accomplished, quite a range of $A$ operators may be considered; for example, powers of the Laplacian, as is considered here.

\section{Admissibility and the Operator Carleson Measure Criterion}

We describe in this section some background material on admissibility and the operator Carleson measure criterion. The reader is referred to $[5,6,13]$ for details.

\subsection{Admissibility}

Suppose that $A$ is the generator of a strongly continuous semigroup $\mathbb{T}=\left(\mathbb{T}_{t}\right)_{t \geq 0}$ on the Hilbert space $V_{0}$. Define the space $V_{-1}$ as the completion of $V_{0}$ with respect to the norm

$$
\|x\|_{-1}=\left\|(\beta I-A)^{-1} x\right\|,
$$

where $\beta \in \rho(A)\left(V_{-1}\right.$ does not depend upon $\left.\beta\right)$. Then $(\beta I-A)^{-1}$ extends to an isomorphism from $V_{-1}$ to $V_{0}$. T extends to a strongly continuous semigroup on $V_{-1}$, whose generator is an extension of $A$ with domain $V_{0}$. The extended semigroup is isomorphic to the initial one. (We will not make notational distinctions for various extension operators.) Let $Z_{1}$ be the Hilbert space obtained by endowing $D\left(A^{*}\right)$ with the norm

$$
\|x\|_{1}=\left\|\left(\beta I-A^{*}\right) x\right\| .
$$

When $V_{0}$ is identified with $V_{0}^{*}$ it follows that $Z_{1}^{*}=V_{-1}$.

Let $U$ be a Hilbert space and suppose $B \in \mathcal{L}\left(U, V_{-1}\right)$. Consider the system

$$
\dot{x}(t)=A x(t)+B u(t), \quad x(0)=x_{0} .
$$

Given any $x_{0} \in V_{0}$ and any $u \in L_{l o c}^{2}([0, \infty), U)$ the unique solution (in $V_{-1}$ ) of $(2.1)$ is given by

$$
x(t)=\mathbb{T}_{t} x_{0}+\Phi_{t} u
$$

where $\Phi_{\tau} u$ is defined by

$$
\Phi_{\tau} u=\int_{0}^{\tau} \mathbb{T}_{r-\sigma} B u(\sigma) d \sigma .
$$


In particular, $x \in C\left([0, \infty), V_{-1}\right)$. It is not necessarily true that $x \in C\left([0, \infty), V_{0}\right)$.

Definition 2.1 With the above notation, let $U$ be a Hilbert space and $B \in \mathcal{L}\left(U, V_{-1}\right)$. Then $B$ is said to be an admissible control operator for $\mathbb{T}$ relative to the space $V_{0}$, if for some $\tau>0$ and any $u \in L^{2}([0, \infty), U)$ we have $\Phi_{\tau} u \in V_{0}$.

If $B$ is admissible then for any $\tau \geq 0, \Phi_{\tau}$ is a bounded linear operator from $L^{2}([0, \infty), U)$ to $V_{0}$ (this follows from the closed graph theorem). In other words, for each $\tau \geq 0$ there is a $k_{\tau} \geq 0$ such that

$$
\left\|\Phi_{\tau} u\right\|_{V_{0}} \leq k_{\tau}\|u\|_{L^{2}} \quad \forall u \in L^{2}([0, \infty), U) .
$$

It follows that given any $x_{0} \in V_{0}$ and any $u \in L_{l o c}^{2}([0, \infty), U)$ the solution of $(2.1)$ has the regularity

$$
x \in C\left([0, \infty), V_{0}\right) .
$$

\subsection{Operator Carleson Measure Criterion}

The Carleson measure criterion of Ho and Russell, [8] and Weiss, [14] provides a necessary and sufficient condition for the admissibility of control operators for diagonal semigroups on $l^{2}$ in the case of scalar inputs. In $[5,6]$ a related condition, called the operator Carleson measure criterion (OCMC), was shown to provide a necessary and sufficient condition for admissibility in the case of $l^{2}$-valued inputs if certain spectral conditions on the semigroup generator are satisfied. We describe below some of the main results of $[5,6]$.

We represent elements of $l^{2}$ as infinite column matrices (of complex numbers) and elements of the dual $l^{2 *}$ as infinite row matrices.

The OCMC applies to systems of the form

$$
\dot{x}(t)=\Lambda x(t)+\widehat{B} u(t),
$$

with input $u \in L^{2}\left([0, \infty), l^{2}\right)$ and where $\Lambda$ and $\widehat{B}$ are represented by infinite matrices. $\Lambda$ is diagonal and its diagonal elements $\lambda_{k}$ (its eigenvalues) are in a left half of the complex plane:

$$
-\lambda_{k} \in \mathbb{C}_{\varepsilon}:=\{s \in \mathbb{C} \mid \operatorname{Re} s>\varepsilon\} \quad \forall k \in \mathbb{N} .
$$

For our purposes we may assume that $\varepsilon$ is some positive number. (Otherwise Definition 2.2 below can be modified accordingly.) Therefore $\Lambda$ generates a strongly continuous diagonal semigroup $\mathbb{T}=\left(\mathbb{T}_{t}\right)_{t \geq 0}$ on $l^{2}$ :

$$
\left(\mathbb{T}_{t} z\right)_{k}=e^{\lambda_{k} t} z_{k}, \quad \forall k \in \mathbb{N},
$$

where $z_{k}$ denotes the $k$-th component of $z \in l^{2}$. The infinite matrix $\widehat{B}$ may be unbounded in the sense that the image of $l^{2}$ is not necessarily contained in $l^{2}$.

To recall the results of $[5,6]$, we need a notation for certain rectangles in $\mathbb{C}$ :

$$
R(h, w)=\{z \in \mathbb{C} \mid 0<\operatorname{Re} z \leq h, w-h \operatorname{Im} z<w+h\},
$$

for any $h+i w \in \mathbb{C}_{0}(i=\sqrt{-1})$. We denote by $b_{k}$ the $k$-th row of $\widehat{B}$. The $b_{k}^{*} b_{k}$ is an infinite matrix of rank one. 
Definition 2.2 The infinite matrix $\widehat{B}$, with rows $b_{k}$, satisfies the operator Carleson measure criterion for the semigroup $\mathbb{T}$ defined by (2.5) if $b_{k} \in l^{2 *}$ for any $k \in \mathbb{N}$ and there is some $M \geq 0$ such that for any $h+i w \in \mathbb{C}_{0}$,

$$
\left\|\sum_{-\lambda_{k} \in R(h, w)} b_{k}^{*} b_{k}\right\|_{\mathcal{L}\left(l^{2}\right)} \leq M h .
$$

The space of infinite matrices which satisfy the operator Carleson measure criterion for $\mathbb{T}$ is denoted by $\operatorname{OCM}(\mathbb{T})$. The following results were proved in [5], [6].

Theorem 2.3 Assume $\mathbb{T}$ is defined by (2.4) and the infinite matrix $\widehat{B}$ represents an admissible control operator for $\mathbb{T}$. Then $\widehat{B} \in O C M(\mathbb{T})$.

Theorem 2.4 Assume $\mathbb{T}$ is defined by (2.4) and $\widehat{B} \in O C M(\mathbb{T})$. Then $\widehat{B}$ represents an admissible control operator for $\mathbb{T}$ if any one of the following conditions hold

(1) $\mathbb{T}$ is invertible (i.e., extends to a group),

(2) $\mathbb{T}$ is analytic,

(3) There are numbers $0, a \leq b$ and $\alpha \geq 0$ such that

$$
a\left|\operatorname{Im} \lambda_{k}\right|^{\alpha} \leq-\operatorname{Re} \lambda_{k} \leq b\left|\operatorname{Im} \lambda_{k}\right|^{\alpha}
$$

Then $\widehat{B} \in O C M(\mathbb{T})$ if and only if $\widehat{B}$ is an admissible control operator for $\mathbb{T}$.

The additional conditions (1), (2) and (3) in Theorem 2.4 are restrictions possible positions of the eigenvalues of the generator $\Lambda$. It is unknown whether Theorem 2.4 remains valid without any such restrictions; see [5, Conjecture 4.4].

\section{Application to damped elastic systems}

Let $\Omega=\{x, y: 0<x<1,0<y<1\}$ and let $\Delta_{0}$ denote the Dirichlet Laplacian operator on the domain $\Omega$. Initially, $\Delta_{0}$ is defined by its variational formulation on the Sobolev space $H_{0}^{1}(\Omega)$ :

$$
\left\langle\Delta_{0} \psi, \phi\right\rangle_{\Omega}=-\int_{\Omega} \nabla \psi \cdot \nabla \phi d \Omega \quad \forall \phi \in H_{0}^{1}(\Omega) .
$$

The form $\langle\cdot, \cdot\rangle$ in (3.1) denotes the duality pairing relative to the pivot space $X_{0}=$ $L^{2}(\Omega)$. It is well-known that $-\Delta_{0}$ is positive, self-adjoint and provides an isomorphism (topologically and algebraically) from $H_{0}^{1}$ to $H^{-1}$. As a consequence, a family of function spaces $X_{a}, a \in \mathbb{R}$ can be constructed (by interpolation) so that $-\Delta_{0}: X_{a} \rightarrow X_{a-1}$ is an isomorphism. For example, since $X_{0}=L^{2}(\Omega)$ we have $X_{1}=H^{2}(\Omega) \cap H_{0}^{1}(\Omega)$, while $X_{-1 / 2}=H^{-1}(\Omega)$. From the spectral theorem all powers of $-\Delta_{0}$ are well-defined and thus for $p>0,\left(-\Delta_{0}\right)^{p}: X_{a+p} \rightarrow X_{a}$ is an isomorphism. 
The control problems in Examples 1 and 2, (for simplicity, with control only on one edge $\Gamma_{0}=\{(x, y): x=0\}$ of the domain) can be written

$$
w_{t t}+2 \gamma\left(\left(-\Delta_{0}\right)^{p}\right)^{\alpha} w_{t}+\left(-\Delta_{0}\right)^{p} w=\delta_{0}^{\prime}(y) f(x, t), \quad(x, y, t) \in \Omega \times \mathbb{R}^{+} .
$$

In terms of the notation in (1.1), we are using $A=\left(-\Delta_{0}\right)^{p}$, where $p=1$ in Example 1 and $p=2$ in Example 2. $B=\delta_{0}^{\prime}(y)$, where $\delta_{0}^{\prime}(y)$ denotes the functional (in $X_{-1}$ ) that assigns to each function in $X_{1}$ the normal derivative on $\Gamma$. Then one has $B: U=L^{2}(0,1) \rightarrow Y_{-1}=X_{-p}$ is continuous. The control function $f$ in (3.2) is assumed to belong to the class $L^{2}\left((0,1) \times \mathbb{R}^{+}\right)$so that $\delta_{0}(y) f(x, t) \in L^{2}\left(0, T ; X_{-1}\right)$. Then a solution of (3.2) is well-defined via the usual variation of constants formula. The representation (3.2) of the problems in Examples 1 and 2 is valid in the sense that the solution of (3.2) (defined by variation of constants) is the same as the transpositional (weak) solution of the boundary value problems in Example 1,2; see Lions and Magenes [11].

As mentioned earlier, it is known that when $\alpha \geq 1 / 2$ the solutions of (3.2) are determined by an analytic semigroup and consequently hypothesis (2) in Theorem 2.4 is satisfied. In the case $\alpha=0$ the associated semigroup is invertible, so that hypothesis (1) of Theorem 2.3 applies. If $0<\alpha<1 / 2$ the results of S. Chen and Triggiani $[2,3]$ show that the associated semigroups are neither analytic nor invertible. We will see however, that hypothesis (3) of Theorem 2.4 is satisfied. In what follows we restrict our interest to the latter case: $0<\alpha<1 / 2$, although the same general approach is valid for if $\alpha=0$ or $\alpha \geq 1 / 2$.

\subsection{Calculation of matrix representation}

Letting $\mathbf{w}=\left(w_{1}, w_{2}\right)^{T}=\left(w, w_{t}\right)^{T}$ we can rewrite $(3.2)$ as

$$
\frac{d \mathbf{w}}{d t}=\mathcal{A} \mathbf{w}+\mathcal{B} f
$$

where

$$
\mathcal{A} \mathbf{w}=\left(\begin{array}{c}
w_{2} \\
-\left(-\Delta_{0}\right)^{p} w_{1}-2 \gamma\left(\left(-\Delta_{0}\right)^{p}\right)^{\alpha} w_{2}
\end{array}\right), \quad \mathcal{B} f=\left(\begin{array}{c}
0 \\
\delta_{0}^{\prime} f
\end{array}\right) .
$$

It is easy to show that $\mathcal{A}: X_{p / 2} \times X_{0} \rightarrow X_{0} \times X_{-p / 2}$ is an isomorphism. Likewise, for any $a \in \mathbb{R}, \mathcal{A}$ can be extended continuously (or restricted) to an isomorphism: $V_{a} \rightarrow V_{a-1}$, where

$$
V_{a}=X_{a p / 2+p / 2} \times X_{a p / 2}
$$

The next task is to compute the matrix representations of $\mathcal{A}$ and $\mathcal{B}$ with respect appropriate bases. For $j, k \in \mathbb{N}$ let $e_{j k}(x, y)=2 \sin j \pi y \sin k \pi x$. Then

$$
\left(-\Delta_{0}\right)^{p} e_{j k}=s_{j k}^{p} e_{j k} ; \quad s_{j k}=\pi^{2}\left(j^{2}+k^{2}\right) .
$$

The eigenvalues $\lambda_{j k}^{+}, \lambda_{j k}^{-}$of $\mathcal{A}$ are given in terms of $s_{j k}$ by

$$
\lambda_{j k}^{ \pm}=-\gamma s_{j k}^{p \alpha} \pm i s_{j k}^{p / 2} \sqrt{1-\gamma^{2} s_{j k}^{p(2 \alpha-1)}} \simeq-\gamma s_{j k}^{p \alpha} \pm i s_{j k}^{p / 2} \text { as }\left|s_{j k}\right| \rightarrow \infty .
$$


(We write $f(s) \simeq g(s)$ as $|s| \rightarrow \infty$ if $f$ and $g$ match to highest order as $|s| \rightarrow \infty$.) Note that

$$
\left(-\operatorname{Re} \lambda_{j k}^{ \pm}\right) \simeq-\gamma\left|\operatorname{Im}\left(\lambda_{j k}^{ \pm}\right)\right|^{2 \alpha} \text { as }\left|\lambda_{j k}^{ \pm}\right| \rightarrow \infty
$$

and consequently the condition (3) of Theorem 2.4 applies.

The eigenfunctions $\Psi_{j k}^{ \pm}$corresponding to the eigenvalues $\lambda_{j k}^{ \pm}$are

$$
\Psi_{j k}^{ \pm}=\frac{1}{\sigma_{j k}}\left(\begin{array}{c}
1 \\
\lambda_{j k}^{ \pm}
\end{array}\right) e_{j k}
$$

where $\sigma_{j k}$ is picked so that the sequence $\left(\Psi_{j k}^{ \pm}\right)$is normalized in the space $V_{a}$. Since $\left|\lambda_{j k}^{ \pm}\right| \simeq s_{j k}$ as $s_{j k} \rightarrow \infty$ we have

$$
\left\|\Psi_{j k}^{ \pm}\right\|_{a}=\left|\sigma_{j k}\right|^{-1}\left(\left\|A^{(a+1) / 2} e_{j k}\right\|_{L_{2}}+\left\|(A)^{a / 2} \lambda_{j k}^{ \pm} e_{j k}\right\|_{L_{2}}\right) \simeq\left|\sigma_{j k}\right|^{-1}\left(2 s_{j k}^{(a p+p / 2)}\right),
$$

and hence

$$
\sigma_{j k}=\left(2 s_{j k}^{p(a+1) / 2}\right)
$$

It can be shown (for example, by a proof similar to one in [7]) that with the above normalization, the eigenfunctions $\left(\Psi_{j k}^{ \pm}\right)$form a Riesz basis for $V_{a}$. This implies that the mapping: $V_{a} \rightarrow l^{2}$ defined by $\sum c_{j k}^{ \pm} \Psi_{j k}^{ \pm} \rightarrow\left(c_{j k}^{ \pm}\right)$is an isomorphism.

If we order the basis $\left(\Psi_{j k}^{ \pm}\right)$as $\left(\Psi_{11}^{+}, \Psi_{11}^{-}, \Psi_{21}^{+}, \Psi_{21}^{-}, \ldots, \Psi_{12}^{+}, \Psi_{12}^{-}, \ldots, \Psi_{13}^{+}, \ldots\right)$ then the matrix representation $A$ of $\mathcal{A}$ with respect to this basis is

$$
A=\operatorname{diag}\left(A_{1}, A_{2}, \ldots\right), \quad \text { where } A_{k}=\operatorname{diag}\left(\lambda_{1 k}^{+}, \lambda_{1 k}^{-}, \lambda_{2 k}^{+}, \lambda_{2 k}^{-}, \ldots\right) .
$$

To calculate the matrix representation $B$ of $\mathcal{B}$ we write

$$
\begin{gathered}
f(x, t)=\sum_{k \in \mathbb{N}} f_{k}(t) \sin k \pi x \\
\delta_{0}^{\prime}(y)=\sum_{j \in \mathbb{N}}\left\langle\delta_{0}^{\prime}(y), \sqrt{2} \sin j \pi y\right\rangle \sqrt{2} \sin j \pi y=2 \sum_{j \in \mathbb{N}} j \pi \sin j \pi y .
\end{gathered}
$$

Therefore

$$
\mathcal{B} f(x, y, t)=\left(\begin{array}{c}
0 \\
\sum_{j, k \in \mathbb{N}} j \pi f_{k}(t) e_{j k}
\end{array}\right)=\sum_{j, k \in \mathbb{N}}\left(b_{j k}^{+} \Psi_{j k}^{+}+b_{j k}^{-} \Psi_{j k}^{-}\right) f_{k}(t),
$$

where $b_{j k}^{ \pm} \simeq \pm \sigma_{j k} j \pi /\left(2 i s_{j k}^{p / 2}\right)$ as $\left|s_{j k}\right| \rightarrow \infty$, and hence

$$
\left|b_{j k}^{ \pm}\right| \simeq j \sigma_{j k} \pi\left(2 s_{j k}^{p / 2}\right)^{-1}=\pi j s_{j k}^{p a / 2} \text { as }\left|s_{j k}\right| \rightarrow \infty .
$$

If we denote $u=\left(f_{1}, f_{2}, \ldots\right)^{T}$ then (3.3) is equivalent to a system of the form (1.1) with

$$
B=\operatorname{diag}\left(B_{1}, B_{2}, \ldots\right), \quad \text { where } B_{k}=\left(b_{1 k}^{+}, b_{1, k}^{-}, b_{2 k}^{+}, b_{2 k}^{-} \ldots\right)^{T} .
$$




\subsection{Simplified form of the OCMC}

Due to the known structure of the eigenvalues, the OCMC can be written in a much simpler form, using the following observations.

First, due to this block-diagonal structure of $B$, the infinite matrices (in the notation of (2.6))

$$
\sum_{-\lambda_{k} \in R(h, w)} b_{k}^{*} b_{k}
$$

are all diagonal, and hence to calculate their norms, one only needs to compute the supremum of the diagonal elements. Secondly, due to the conjugate symmetry in the eigenvalues, it will be enough to check the OCMC for $w>0$. Furthermore, since there are only finitely many near the origin, it is enough to check the OCMC as $w \rightarrow+\infty$. Thus, for example, $\operatorname{Im} \lambda_{j k}^{ \pm} \simeq s_{j k}^{p / 2}$ as $\left|\lambda_{j k}^{ \pm}\right| \rightarrow \infty$. Lastly, due to (3.4) we may assume the $h$ in $R(h, w)$ is algebraically related to $w$ by

$$
h=w^{2 \alpha} .
$$

This is due to the fact that taking $h$ of smaller order causes the rectangles to eventually miss all the eigenvalues (and hence trivially satisfy the OCMC), while $h$ of larger order is suboptimal due to the fact that for sufficiently large $w_{0}$, a rectangle with corresponding $h_{0}$ of larger order than in (3.6) can be replaced by two or more (say $K)$ smaller disjoint rectangles $R\left(h_{1}, w_{1}\right), R\left(h_{2}, w_{2}\right)$, etc. with $h_{k}=w_{k}^{2 \alpha}$. Then, denoting $\mu(R(h, w))$ the left hand side of $(2.6)$, one has $\mu\left(R\left(h_{0}, w_{0}\right)\right) \leq \mu\left(R\left(h_{1}, w_{1}\right)\right)+$ $\mu\left(R\left(h_{2}, w_{2}\right)\right)+\cdots+\mu\left(R\left(h_{K}, w_{K}\right)\right)$ so that if $\mu\left(R\left(h_{j}, w_{j}\right)\right) \leq M_{j} h_{j}, j=1,2, \ldots K$, then we also have

$$
\mu\left(R\left(h_{0}, w_{0}\right)\right) \leq \sum_{j=1}^{K} M_{j} h_{j} \leq\left(\operatorname{Max}_{j=1,2, \ldots K} M_{j}\right) h_{0} .
$$

Thus the $M$ (in (2.6)) associated with the larger order rectangle is bounded by the $M$ 's associated with the smaller rectangles. Hence it is enough to check only rectangles $R(h, w)$ with $h=w^{2 \alpha}$.

It follows that an equivalent formulation of the OCMC is

$$
\sup _{k \in \mathbb{N}, w>1} \sum_{j: w \leq s_{j k}^{p / 2} \leq w+h}\left|b_{j k}^{ \pm}\right|^{2} \leq M h ; \quad h=w^{2 \alpha} .
$$

For fixed $k$ let $J=\left\{j: w \leq s_{j k}^{p / 2} \leq w+h\right\}$ and let $j_{0}, j_{1}$ denote the minimal and maximal elements of $J$. (We only need to consider the cases where $J$ is nonempty.) From (3.6) the growth of the size of the intervals $[w, w+h]$ as a function of $w$ is sublinear. It follows that for any number $s$ in this interval, there exists $C>0$ so that $w \leq s \leq C w$. Thus

$$
\sum_{j: w \leq s_{j k}^{p / 2} \leq w+h}\left|b_{j k}^{ \pm}\right|^{2} \simeq \pi^{2} \sum_{j: w \leq s_{j k}^{p / 2} \leq w+h} j^{2} s_{j k}^{p a} \equiv w^{2 a} \sum_{j=j_{0}}^{j_{1}} j^{2}
$$


where the symbol $\equiv$ means that each side is bounded by some fixed multiple (as $w \rightarrow \infty$ ) of the other. Thus, referring to (2.6) (divided through by the $h$ ), an equivalent form of the $\mathrm{OCMC}$ is

$$
\sup _{w>1, k \in \mathbb{N}} w^{2 a-2 \alpha} \sum_{j=j_{0}}^{j_{1}} j^{2}<\infty .
$$

For optimality of (3.7) we seek to find $a$ so that the LHS of (3.7) is finite but nonzero.

\subsection{The case $p=1$}

Let us now specialize to the case $p=1$. We have from the definition of set $J$

$$
w^{2} \leq \pi^{2}\left(j_{0}^{2}+k^{2}\right) \leq \pi^{2}\left(j_{1}^{2}+k^{2}\right) \leq(w+h)^{2},
$$

so that $\pi^{2}\left(j_{1}^{2}-j_{0}^{2}\right) \leq 2 w h+h^{2}=2 w^{1+2 \alpha}+w^{4 \alpha} \leq 3 w^{1+2 \alpha}$. We thus have the estimate

$$
j_{1} \leq j_{0}(1+R)^{1 / 2}, \quad \text { where } R=\left(3 / \pi^{2}\right) w^{1+2 \alpha} / j_{0}^{2} .
$$

We find that

$$
\begin{aligned}
& \sum_{j=j_{0}}^{j_{1}} j^{2}=\frac{j_{1}^{3}-j_{0}^{3}}{3}+\frac{j_{0}^{2}+j_{1}^{2}}{2}+j_{1}-j_{0} \leq \frac{4}{3}\left(j_{1}^{3}-j_{0}^{3}\right)+w^{2} \\
& \leq \frac{4}{3} j_{0}^{3}\left((1+R)^{3 / 2}-1\right)+w^{2} \leq 2 j_{0}^{3}\left(R+R^{3 / 2}\right)+w^{2} \\
& \leq j_{0} w^{1+2 \alpha}+w^{3(1+2 \alpha) / 2}+w^{2} \leq w^{2+2 \alpha}+w^{3(1+2 \alpha) / 2}+w^{2}
\end{aligned}
$$

Thus, by (3.7), the OCMC will hold if the following three conditions hold:
(i) $2 a-2 \alpha+2+2 \alpha \leq 0$,
(ii) $2 a+2 \alpha+3 / 2+3 \alpha \leq 0$,
(iii) $2 a-2 \alpha+2 \leq 0$.

Therefore admissibility holds if $a \leq-1$. For necessity, note that the condition $a \leq-1$ is required by the inequality (i) above. Furthermore it is simple to check that all inequalities leading to (i) become sharp if one takes $k$ bounded and $\alpha<1 / 2$ (so that $R$ in (3.8) is small). Therefore $a \leq-1$ is optimal for the problem (3.2) for $0<\alpha<1 / 2$. This implies (due to the previously discussed Riesz basis isomoprhism) that the solutions of (3.2) satisfy the optimal regularity

$$
w \in C\left([0, \infty), L^{2}(\Omega)\right) \cap C^{1}\left([0, \infty), H^{-1}(\Omega)\right),
$$

which is in agreement with the optimal results for the undamped case (see [10]). Since the problem in Example 1 can be represented in the form (3.2) if $0 \leq \alpha \leq 1 / 4$, the regularity in (3.9) also applies to Example 1 for $0 \leq \alpha \leq 1 / 4$.

\subsection{The cases $p>1$}

We find more interesting behavior when $p>1$. Nevertheless, most of the steps are the same and so we follow the same steps and notation used for the case $p=1$, only indicating the differences. 
Set $J$ is defined by $J=\left\{j: w \leq s_{j k}^{p / 2} \leq w+h\right\}$ so that

$$
w^{2 / p} \leq \pi^{2}\left(j_{0}^{2}+k^{2}\right) \leq \pi^{2}\left(j_{1}^{2}+k^{2}\right) \leq(w+h)^{2 / p} .
$$

Thus we have $\pi^{2}\left(j_{1}^{2}-j_{0}^{2}\right) \leq(w+h)^{2 / p}-w^{2 / p} \simeq\left(w^{2 / p} h / w\right) f^{\prime}(1)$ as $w \rightarrow \infty$ where $f(t)=t^{2 / p}$. Therefore we obtain $\left(j_{1}^{2}-j_{0}^{2}\right) \leq C w^{2 / p+2 \alpha-1}$ where $C$ is independent of $w$. It follows we again obtain an estimate like (3.8):

$$
j_{1} \leq j_{0}(1+R)^{1 / 2}, \quad \text { where } R=C w^{2 / p+2 \alpha-1} / j_{0}^{2} .
$$

From the same chain of inequalities following (3.8) we obtain

$$
\begin{aligned}
\sum_{j=j_{0}}^{j_{1}} j^{2} \leq 2 j_{0}^{3}\left(R+R^{3 / 2}\right)+w^{2 / p} \leq & C\left\{j_{0} w^{2 / p+2 \alpha-1}+w^{3 / p+3 \alpha-3 / 2}+w^{2 / p}\right\} \\
& \leq C\left\{w^{3 / p+2 \alpha-1}+w^{3 / p+3 \alpha-3 / 2}+w^{2 / p}\right\} .
\end{aligned}
$$

Hence from (3.7) the OCMC holds if the following three conditions hold:

$$
\begin{aligned}
& 2 a-2 \alpha+3 / p+2 \alpha-1=2 a+3 / p-1 \leq 0 \\
& 2 a-2 \alpha+3 / p+3 \alpha-3 / 2=2 a+\alpha+3 / p-3 / 2 \leq 0 \\
& 2 a-2 \alpha+2 / p \leq 0 .
\end{aligned}
$$

One sees that for $\alpha$ near zero inequality (iii) implies the other two, while for $\alpha \geq(p-1) / 2 p$ the first implies the other two. Retracing the inequalities involved, one again sees that all the inequalities involved in obtaining (i) and (iii) are reversible (up to a multiplicative constant) for $k$ bounded. Hence $\widehat{a}$, the optimal value of $a$ is given by

$$
\widehat{a}=\left\{\begin{array}{lll}
\alpha-1 / p & \text { if } & 0<\alpha \leq \frac{p-1}{2 p} \\
1 / 2-3 / 2 p & \text { if } & 1 / 2>\alpha \geq \frac{p-1}{2 p} .
\end{array}\right.
$$

In Example $2 p=2$ and hence the system is admissible if

$$
a \leq \widehat{a}=\min \{-1 / 4, \alpha-1 / 2\} .
$$

It follows that solutions of (3.2) (and hence also the boundary value problem in Example 2) have the regularity

$$
w \in C\left([0, \infty), X_{\widehat{a}+1}\right) \cap C^{1}\left([0, \infty), X_{\widehat{a}}\right) .
$$

It is interesting to note that the regularity improvement due to the damping ceases when $\alpha=1 / 4$. This is perhaps surprising since analyticity is obtained only when $\alpha=1 / 2$. The same general behavior is seen for all values of $p>1$.

\section{Conclusion}

In this article we have indicated an approach based upon the Operator Carleson Measure Criterion to obtain sharp regularity results for systems of the form (1.1). Such systems include some relevant damped elastic systems such as those mentioned 
in Examples 1 and 2. However this method is directly applicable only to special domains, such as rectangles or parallelpipeds, since one must be able to calculate Fourier coefficients corresponding to the control input operator.

The analysis here indicates that for $p=1$ there is no increase in regularity as the damping exponent $\alpha$ is increased. On the other hand, for all $p>1$ there is a linear increase in regularity up to some critical $\alpha_{c}$, after which there is no further increase in regularity. It is particularly interesting that analyticity occurs (for all $p$ ) when $\alpha=1 / 2$, nevertheless $\alpha_{c}$ can assume any value between 0 and $1 / 2$.

\section{References}

[1] G. Chen, D.L. Russell, A mathematical model for linear systems with structural damping, Quarterly of Applied Mathematics 39, 1982. pp. 433-454.

[2] S. Chen, R. Triggiani, Proof of extensions of two conjectures on structural damping for elastic systems, Pacific J. Math. 136, 1989. pp. 15-55.

[3] S. Chen, R. Triggiani, Gevrey class semigroups arising from elastic systems with gentle dissipation: The case $0<\alpha<1 / 2$, Proc. AMS 110, 1990. pp. 401-415

[4] S. Chen, R. Triggiani, Characterizations of domains of fractional powers of certain operators arising in elastic systems, and applications, JDE 88, 1990. pp 279-273

[5] S. Hansen, G. Weiss, The operator Carleson measure criterion for admissibility of control operators for diagonal semigroups on $l^{2}$, Systems and Control Letters 16, 1991. pp 219-227

[6] S. Hansen, G. Weiss, New results on the operator Carleson measure criterion, IMA J. Math. Control Inform 14, 1997. pp 3-32

[7] S. Hansen, Exponential energy decay in a thermoelastic rod, J. Math. Anal. Appl. 167, 1992. pp 429-442

[8] L.F. Ho, D.L. Russell, Admissible input elements for systems in Hilbert space and a Carleson measure criterion, SIAM J. Control \& Optim. 21, 1983. pp 614640

[9] I. Lasiecka, J.L. Lions, R. Triggiani, Non homogeneous boundary value problems for second order hyperbolic operators, J. Math. Pures et Appl. 65, 1986. pp 149192

[10] I. Lasiecka and R. Triggiani, Regularity theory for a class of nonhomogeneous Euler-Bernoulli equations: a cosine operator approach, Topics in mathematical analysis. pp 623-657, 1989. Ser. Pure Math 11 World Sci. Publ, Teaneck, NJ

[11] J.-L. Lions and E. Magenes, Nonhomogeneous Boundary Value Problems and Applications I. Springer-Verlag. New York , 1970

[12] R. Triggiani, Regularity of some structurally damped problems with point control and with boundary dissipation, JMAA 161, 1991. pp 299-331 
[13] G. Weiss, Admissibility of unbounded control operators, SIAM J. Control \& Optim. 27, 1989. pp 527-545

[14] G. Weiss, Admissibility of input elements for diagonal semigroups on $l^{2}$, Systems \& Control Letters 10, 1988. pp 79-82 